\title{
Detection of Abnormalities in Digital Mammogram Images by Analysing Optical Parameters
}

\author{
R. J. Hemalatha, A. Akalya, R. Jeyanthi, T.R.Thamizhvani, Josephin Arockia Dhivya
}

\begin{abstract}
Breast cancer is one of the major lifethreatening cancer that tremendously affects women. According to the survey, the mortality rate of females is increasing due to the belated detection of breast cancer. In recent years, Mammograms plays a vital role in breast cancer detection. In this study, an algorithm is used to detect the microcalcification of the mammogram by calculating its optical characteristics. The considered optical characteristics are the reflection coefficient, absorption coefficient, and transmission coefficient are calculated for the binned mammogram digital images are calculated. The total conservation of energy condition, $R C+T C$ $+A C=1$ is satisfied with the obtained results. For each image, statistical features are extracted, analyzed and are classified with the help of SVM and ANN classifier. This algorithm is verified with about $30 \%$ of MIAS database images. This proposed algorithm acts as a good classifier for the detection of microcalcification thereby considering three optical characteristics.
\end{abstract}

Keywords: Digital Mammogram Images, RoI (Region of Interest) optical Characteristics, classifier.

\section{INTRODUCTION}

$\mathrm{B}_{\text {reast cancer is the leading cause of death for }}$ women worldwide. Breast cancer can be detected through various imaging techniques such as Mammography, Magnetic Resonance Imaging (MRI), and Ultrasound. However, mammography acts as an effective tool for breast cancer detection. A mammogram is an $\mathrm{x}$-ray image that provides soft tissue images where it is convenient in display and is inexpensive. The major abnormalities related to the breast are masses and calcifications. Masses are less vulnerable than calcifications. Calcifications can be further classified as macro and microcalcifications. Macrocalcifications are large calcium deposits in the breast tissue formed due to aging. However, a small number of calcium deposits in the breast tissue can lead to the most vulnerable form of cancer called microcalcification.

Revised Manuscript Received on November 25, 2019.

* Correspondence Author

*R.J.Hemalatha, head \& Assistant Professor -Department of Biomedical Engineering,Vels Institute of Science, Technology \& Advanced Studies, Chennai, India. Email:hodbiomedical.se@ velsuniv.ac.in

A.Akalya-Student -Department of Biomedical ,Engineering,Vels Institute of Science, Technology \& Advanced Studies, Chennai, India.

R.Jeyanthi, Student -Department of Biomedical Engineering,Vels Institute of Science, Technology \& Advanced Studies, Chennai, India.

T.R.Thamizhvani, Assistant Professor -Department of Biomedical Engineering,Vels Institute of Science, Technology \& Advanced Studies, Chennai, India. Email: thamizh.se@ velsuniv.ac.in

Josephin Arockia Dhivya, Assistant Professor Department of Biomedical Engineering,Vels Institute of Science, Technology \& Advanced Studies, Chennai, India. Email: a.dhivya.se@ velsuniv.ac.in
Both masses and microcalcifications are further classified as benign and malignant based on their size, shape, margins. Malignant cancers are more dangerous than benign cancers. Various image processing techniques are useful for the detection of microcalcification and the classification of cancer as benign and malignant using their structural features. This paper proposes the phenomenon related to the classification of microcalcification by the calculation of the optical characteristics of the identified region of interest in the microcalcification. The calculation of the reflection coefficient, transmission coefficient and absorption coefficient of a digital mammogram image was performed for the preprocessed input mammogram images. Finally, the features that are extracted from the digital mammogram images are analyzed and are classified as normal and abnormal cancerous tissue by using the $\mathrm{k}$ Nearest Neighbour (KNN) classifier, Naïve Bayes and Support Vector Machine (SVM) classifier. The statistical parameters are also observed for the digital mammogram images. The parameters considered are mean, median, mode, skewness, kurtosis, min, max, area and standard deviation.

\section{LITERATURE SURVEY:}

In this study it is required to evaluate a mammography image, considering the recent inventions of many scanning and $\mathrm{x}$-ray techniques. The complexity of using a mammography image to detect the microcalcification is found either in small clusters or scattered particles. The optical characteristics are very important to detect microcalcification, thus reflection, absorption and transmission coefficient are considered for the evaluation of optical characteristics in breast imaging.

As Electromagnetic waves propagate from one homogeneous medium to another, they experience a change of the wave impedance in the interface. The impedance mismatch gives the reflection, absorption, and transmission of electromagnetic waves. In the case of non-homogeneous media(human body), it is complex to analyze the behavior of electromagnetic waves. The reflection and transmission coefficients vary from tissue to tissue and they are dependent on permittivity, conductivity, conductivity, and frequency. So it is necessary to study the reflection and transmission coefficients of biological tissue.[5]

Jeba.et.al [7] utilizes sono mammogram for identification of cyst through optical characteristics. For acoustic impedance, if there is a large mismatch, then a large proportion of the wave is reflected, which is a strong echo that produces a bright image on the display, like bone and muscle or air and soft tissue. If there is a small mismatch, then a small proportion of wave is reflected. Remaining is transmitted across the interface. The reflection coefficient is calculated for the $\mathrm{ROI}$ and it is mapped with the

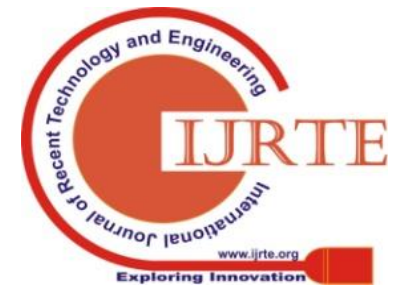


range of mass density of the mammogram image.

Suvithra.et.al [8] proposed a system for detection of the size of microcalcification in embedded systems through the study of hardware implementation. A linear mapping between the ranges of reflection coefficient to mass density is done to confirm the occurrence of microcalcification. Straight mapping of reflection coefficient with mass density is anticipated as 3D and at the same time, the span of first and second level binning are figured to infer the size of the microcalcification. This procedure is executed on hardware to make it more commercial for the common people to distinguish the disease at a beginning period.

Jothilakshmi.et.al [9] proposes a combination of pattern recognition of a phenomenon related to microcalcification. From the RoI, statistical features are extracted, and by using SVM, fuzzy k-means, and C-mean clustering, the identified pattern is classified. By correlating the reflection coefficient and mass density values of the binned image, the prediction class to categorize the considered mammogram image (malignant and normal) was also achieved with better classification accuracy. The 3D view of the projected lesion part and their angle rotation is a user-friendly approach, which could be easily understood by radiologists, physicians, and even by the patients.

Sharkawy.et.al [20] proposes a method to determine the optical properties of breast cancer in the detection of malignant and non-malignant breast tissue. The propagation of light in a medium is described by its optical properties. The fundamental optical properties of interest are the absorption coefficient ( $\mu \mathrm{a})$, scattering coefficient $(\mu \mathrm{s})$, total attenuation coefficient $(\mu \mathrm{t})$, scattering anisotropy $(\mathrm{g})$, reduced scattering coefficient $(\mu \mathrm{s})^{\prime}$, effective attenuation coefficient ( $\mu$ eff), and the tissue refractive index (n). By measuring the optical properties of the non-malignant and malignant breast tissues using optical spectroscopy, the average absorption coefficient for normal breast tissue samples at $633 \mathrm{~nm}$ is $23(\mathrm{~cm}-1)$ with a standard deviation of 0.091 . The diffuse reflectance and depth of light penetration in a medium are dependent on the absorption and scattering coefficients.

Elizabeth.et.al [15] proposes a method for feature extraction of mammogram images to build a ComputerAided Diagnosis (CADx) system to discriminate between normal, benign and malignant masses. The feature extraction is based on Multidimensional Complete Ensemble Empirical Mode Decomposition with Adaptive Noise(MCEEMDAN)which decomposes a given image into frequency components called intrinsic mode functions(IMFs). The texture is an important characteristic that helps to discriminate between normal and lesion regions in mammograms. Artificial Neural Networks (ANN) was used as a classifier and the maximum classification accuracy of $96.7 \%$ with $95.5 \%$ sensitivity and $93.3 \%$ specificity is achieved.

Fraschini.et.al [23] utilizes a system to consider only small 1D signals crossing the region of interest, allowing one to drastically reduce the amount of data to be processed. A very simple novel computer-aided diagnosis (CAD) scheme of breast cancer in digitized mammograms. The novelty of this system is mainly focused on the significant dimensionality reduction that permits drastically decreasing the amount of data to characterize the regions of interest. It has been shown, in mammographic mass classification, that the wavelet transform and neural network do not lose their power in discriminating between benign/malignant regions also if applied on 1D signals extracted from the suspicious areas.

Shafiullah.et.al [10] proposed a new region-based active contour method is proposed for mammogram image segmentation. It introduces a new shifted Heaviside SPF function, which uses a global fitted image restricted with mask term to enforce level set evolution inwards. If the mask term set to 1 then it starts modulating its signs inside and outside of regions of interest. The proposed method has accurately segmented abnormal regions from the miniMIAS database. It presents a fast and effective method for detecting high-intensity regions in mammograms.

Hayati.et.al [17], a novel method for classifying salient dense regions in mammograms is proposed. It involves the detection of threshold based regions, elimination with the decision tree process, computing features and minimum nesting depths of the region of interests and finally classification by using Support Vector Machines (SVM ). Experimental results depicts that the proposed method achieves good performance for detecting masses in the mammogram.

Narain.et.al [25]proposed few novel local binary textural patterns for classification of mammograms which was found to have a consistent accuracy rate.

Using $\mathrm{K}$ means segmentation algorithm and Gray level cooccurrence matrices for extracting textural features of the segmented area.

\section{METHODOLOGY}

The Digital Mammogram Images are used to detect breast cancer and can determine the microcalcification. The digital mammogram images have been enhanced and the Region Of Interest (RoI) i.e. the lesion part is segmented for the feature extraction. The extracted feature values are used to classify as normal and abnormal cancerous tissue by using different classifiers. The block diagram shown in the Fig 1 depicts the flow of the proposed work.

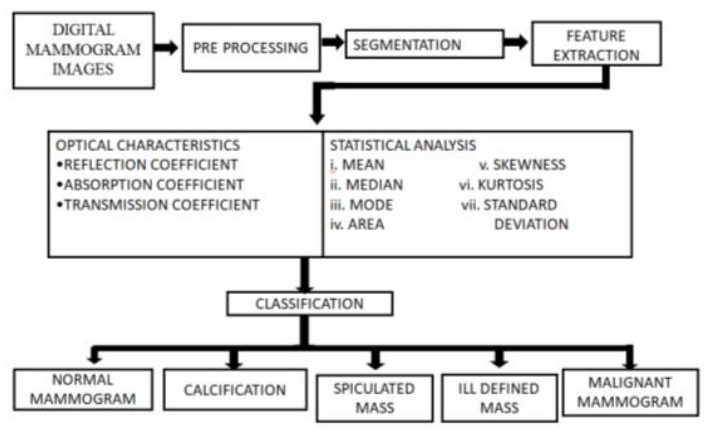

Fig 1: Block Diagram

\section{A. Image acquisition:}

In this paper, the Digital Mammogram Images are obtained from MIAS(online) database. They contain normal and abnormal mammogram images. Benign and malignant images have been reported in the abnormal image category. In total, the database contains 322mammogram images. About $30 \%$ of MIAS database

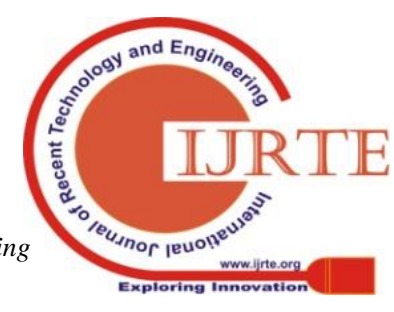


images are utilized for the detection and classification of microcalcification in mammogram images.

\section{B. Preprocessing:}

Preprocessing is used to increase the contrast of the mammographic images so that the difference in intensity level can be computed easily for the detection of masses. Conventional mammograms are noisy, low contrast, and blurred images. Mammogram enhancement is necessary to highlight specific features of images. After the digital mammogramimages has been obtained, various methods of processing can be applied to the images to segment the lesion part from the background tissue for further analysis.

\section{Segmentation:}

The preprocessed image has been initially binned to segment the lesion part i.e., RoI from the Digital Mammogram Image. Binning is a technique that divides the images into sub-regions, and it is essentially useful to move closer to the lesion part and further perform the analysis only with the selected sub-regions.

\section{Binning:}

As per the characteristics of a microcalcification, it may be distributed into more than one bin. In image processing, binning is a technique that combines a cluster of pixels in a single pixel. In first-level binning, each bin presents its matrix of $256 \times 128$ with three rows and four columns bin image. Therefore, in second-level binning, to obtain the optical characteristics more than one bin needs to be analyzed. The bin, which contains the RoI, is identified and again subjected to further binning with two rows and two columns. In the second-level binning, each bin has its matrix of $128 \times 64$

\section{E. Feature extraction:}

For the considered RoI segment of the Digital Mammogram Image, the optical characteristics such as reflection coefficient (RC), absorption coefficient (AC) and transmission coefficient (TC) are calculated. The statistical features that are extracted from the images are mean, median, mode, min, max, skewness, kurtosis, integral density, and standard deviation.

Mean is the average value of the signal. Median is the value separating the higher half from the lower half of the data sample. A mode is the number that occurs most often in a data sample. Min and max are the minimum and maximum amplitude values of the signal. Kurtosis is the measure of tailedness of the distribution of the signal. Skewness is the measure of asymmetry distribution of the signal. Integrated density is the sum of the values of the pixels in the image or selection. This is equivalent to the product of Area and Mean GrayValue. The standard deviation is a statistic that measures the dispersion of a data sample relative to its mean and is calculated as the square root of the variance.

\section{Reflection coefficient:}

The intensity of the reflected wave is described by the reflection coefficient and every tissue interface consists of its own reflection coefficient . The reflection coefficient is a parameter that defines how much of an electromagnetic wave(light) is reflected by an impedance discontinuity in the transmission medium. The reflection coefficient is the ratio of the reflected wave and the incident wave.

$$
\begin{array}{r}
R C=\frac{\text { REFLECTED } W A V E}{\text { INCIDENT } W A V E} \\
\tau=\frac{Z_{B}-Z_{A}}{Z_{B}+Z_{A}}
\end{array}
$$

\section{Absorption coefficient}

The electromagnetic waves are not only reflected from the surface it is also absorbed by the surface. The light energy is absorbed by the molecules of a body and is then transformed into kinetic energy. The rise in movement of the molecules produces heat which is radiated to the surroundings. According to Beer Lambert's law, the absorption coefficient describes the intensity attenuation of the light passing through a material,

$$
A C=\log 10(1 / R)
$$

\section{Transmission coefficient}

The transmission coefficient depicts how much of an electromagnetic wave(light) passes through a surface or an optical element. Due to the conservation of energy law the energy that would be transmitted through the boundary(TI) would represent all the energy from the incident sound wave which was not reflected The transmission coefficient is used to describe the amplitude, intensity or total power of a transmitted wave relative to an incident wave.

The transmission coefficient is the ratio of transmitted wave and incident wave

$$
\begin{array}{r}
T C=\frac{\text { TRANSMISSION WAVE }}{\text { INCIDENT WAVE }} \\
T=\frac{2 n_{1} \cos }{n_{1} \cos +n_{2} \cos }
\end{array}
$$

\section{F. Classification:}

\section{Support vector machine classifier:}

In machine learning, Support Vector Machines are supervised learning models that are associated with learning algorithms used to analyze data for classification and regression analysis. A support vector machine constructs a hyperplane in a high or infinite-dimensional space for classification, regression or other tasks like outlier detection. The average of the feature values obtained is allowed for classification using a linear SVM classifier. 


\section{K nearest neighbour classifier:}

$\mathrm{K}$ nearest neighbors is a simple algorithm that can store all available cases and classifies new cases based on a similarity measure (e.g., distance functions). KNN has been used in statistical estimation and pattern recognition as a non-parametric technique. The algorithm is versatile and it can also be used for classification and regression.

\section{Naive bayes classifier:}

A Naive Bayes classifier belongs to the family of a probabilistic machine learning model that is used for the classification task. They are fast and easy to implement with strong independence assumptions between the features.

\section{RESULTS AND DISCUSSION:}

The mammogram images obtained from the MIAS database are initially preprocessed using different image processing techniques. The processed image is subjected to image binning process to segment the region of interest. The mammogram image obtained from MIAS database is shown in Fig 2. By preprocessing method the lesion part is detected and it is shown in Fig 3, which is the considered input image for the calculation of optical characteristic
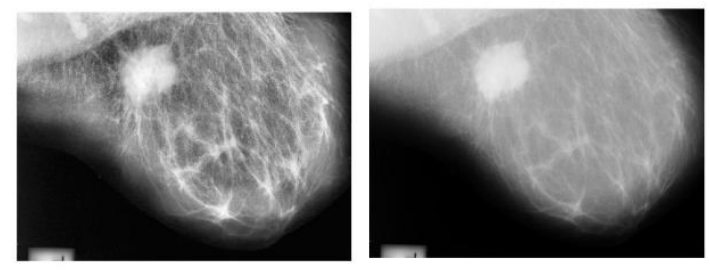

Fig 2: MIAS image

Fig 3: Preprocessed image

The MIAS database images are initially preprocessed and are then segmented to separate the lesion part from the surrounding tissue. For this purpose, binning method is used to segment the lesion tissue. The bin, which contains the RoI, is identified and it is again subjected to second level binning. The first and second level binning are shown in Fig 4 and 5 .

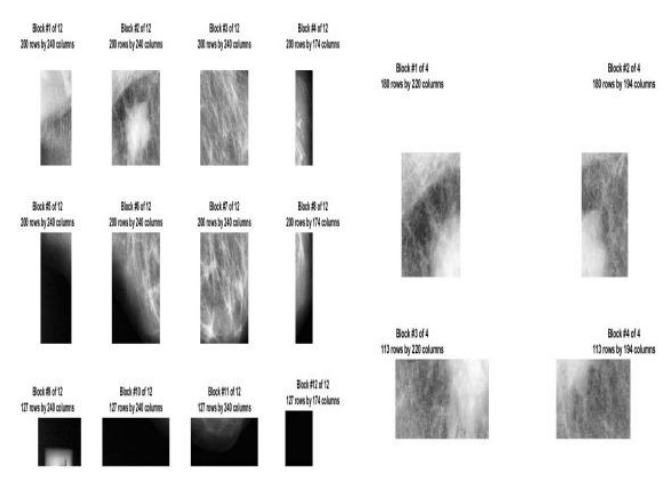

Fig 4: First bin image Fig 5: Second bin image

This paper focuses to satisfy the condition that is the total sum of transmittance, reflectance and absorbance should be equal to one. This is a borrowed concept from the conservation of total energy in a solar radiation beam. The amount of solar radiation transmitted, absorbed and reflected is dependent upon the wavelength the radiation, the incident angle and the optical properties of the material.

The reflection, absorption and transmission coefficient for the considered RoI is tabulated for normal images is tabulated in table 1. And for abnormalities like calcification, spiculated masses, ill-defined masses, malignant images the observed $\mathrm{RC}$, TC and $\mathrm{AC}$ values are tabulated in table 2, 3, 4 and 5 respectively.

Table 1: Physical analysis for normal images

\begin{tabular}{|c|c|c|c|c|}
\hline S.no & Images & RC & TC & AC \\
\hline 1 & Normal & 0.698 & 0.612 & 0.156 \\
\hline 2 & Normal & 0.721 & 0.634 & 0.241 \\
\hline 3 & Normal & 0.734 & 0.596 & 0.213 \\
\hline 4 & Normal & 0.671 & 0.629 & 0.234 \\
\hline 5 & Normal & 0.651 & 0.643 & 0.156 \\
\hline
\end{tabular}

Table 2: Physical analysis for Calcification images

\begin{tabular}{|c|c|c|c|c|}
\hline S .no & Images & RC & TC & AC \\
\hline 1 & Calcification & 0.556 & 0.532 & 0.321 \\
\hline 2 & Calcification & 0.605 & 0.498 & 0.343 \\
\hline 3 & Calcification & 0.628 & 0.412 & 0.428 \\
\hline 4 & Calcification & 0.612 & 0.409 & 0.376 \\
\hline 5 & Calcification & 0.641 & 0.512 & 0.328 \\
\hline
\end{tabular}

Table 3: Physical analysis for spiculated masses

\begin{tabular}{|c|c|c|c|c|}
\hline S. no & Images & RC & TC & AC \\
\hline 1 & Spiculated masses & 0.624 & 0.414 & 0.325 \\
\hline 2 & Spiculated masses & 0.616 & 0.406 & 0.341 \\
\hline 3 & Spiculated masses & 0.564 & 0.524 & 0.387 \\
\hline 4 & Spiculated masses & 0.598 & 0.543 & 0.335 \\
\hline 5 & Spiculated masses & 0.624 & 0.52 & 0.365 \\
\hline
\end{tabular}

Table 4: Physical analysis for ill-defined massed

\begin{tabular}{|c|c|c|c|c|}
\hline S. no & Images & RC & TC & AC \\
\hline 1 & ill-defined masses & 0.614 & 0.425 & 0.454 \\
\hline 2 & ill-defined masses & 0.543 & 0.434 & 0.543 \\
\hline 3 & ill-defined masses & 0.568 & 0.467 & 0.487 \\
\hline 4 & ill-defined masses & 0.623 & 0.498 & 0.365 \\
\hline 5 & ill-defined masses & 0.619 & 0.413 & 0.378 \\
\hline
\end{tabular}

Table 5: Physical analysis for malignant images

\begin{tabular}{|c|c|c|c|c|}
\hline S. no & Images & RC & TC & AC \\
\hline 1 & Malignant & 0.612 & 0.408 & 0.431 \\
\hline 2 & Malignant & 0.543 & 0.432 & 0.423 \\
\hline 3 & Malignant & 0.626 & 0.416 & 0.402 \\
\hline 4 & Malignant & 0.632 & 0.437 & 0.394 \\
\hline 5 & Malignant & 0.643 & 0.425 & 0.376 \\
\hline
\end{tabular}

For normal mammogram images, the reflection, transmission, and absorption coefficient are found to be 0.7 , 0.6 and 0.2 respectively, whereas for abnormal mammogram images the reflection, transmission, and absorption coefficient are found to vary in the range. For the reflection coefficient, the range varies below 0.7 that is 0.5 and 0.6 . In the transmission coefficient, the range varies below 0.6 which is 0.4 and 0.5 and in the absorption coefficient, the range varies above 0.2 that is 0.4 and 0.3 .

It is noted that for both normal and abnormal images are satisfied with the condition

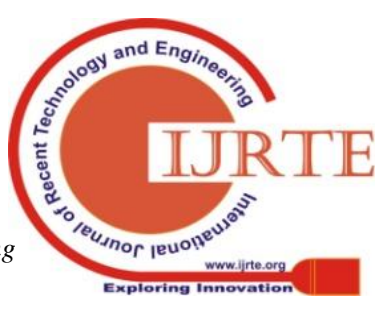


for total conservation of energy that is $\mathrm{RC}+\mathrm{TC}+\mathrm{AC}=1$. In abnormal mammogram images, the reflection and transmission coefficient values are lesser than the normal mammogram images, whereas in the absorption coefficient the values are found to be higher than the normal mammogram images which shows the presence of microcalcification in the digital mammogram images.

The statistical parameters considered for the digital mammogram images are mean, medial, mode, min, max, standard deviation, kurtosis and area. For each mammogram images the statistical features are obtained. The observed values are tabulated in the table 6.

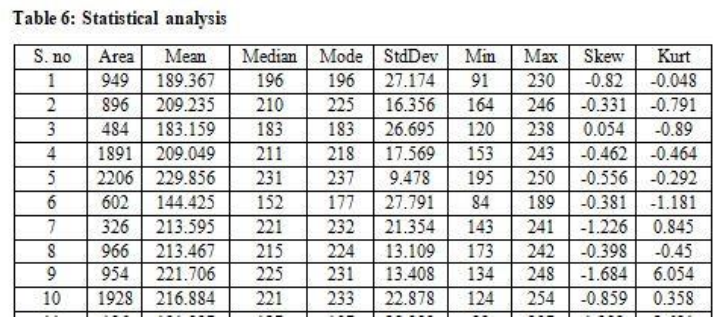

Based on the condition for total conservation of energy, the results depicts that on considering three optical characteristics such as the reflection, absorption and transmission coefficients, the normal and abnormal mammogram images can be classified. Here for the normal mammogram images, the sum of reflection, transmission and absorption coefficients is almost equal to one and for abnormal mammogram images, the absorption coefficient is found to be lesser than the absorption coefficient of normal images, where it also satisfies the condition for total conservation of energy. Thus the proposed algorithm is found to be effective in classification for normal and abnormal mammogram images. The prediction class for the respective optical characteristics is evaluated and it is tabulated in table 7. It is observed that this algorithm gives better classification accuracy for normal and abnormal digital mammogram images.

Table 7: Prediction class

\begin{tabular}{|c|c|c|}
\hline S. No & Classifier & Accuracy \\
\hline 1. & SVM & $97.5 \%$ \\
\hline 2. & KNN & $92.5 \%$ \\
\hline 3. & Naive Bayes & $95 \%$ \\
\hline
\end{tabular}

In comparison with the existing classification techniques, the proposed methodology gives better classification accuracy by considering three optical characteristics of the diagnostic mammograms in correlation with the condition of total conservation of energy.
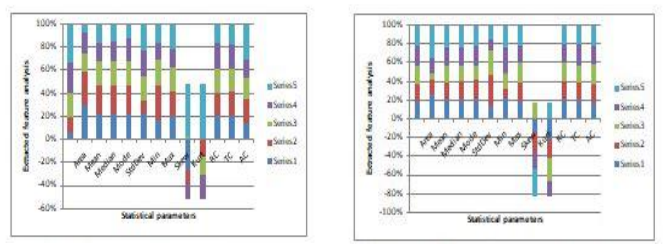

Fig 6: Statistical analysis of normal and abnormal mammogram images

\section{CONCLUSION:}

In this paper, the tumor detection in a digital mammogram is analyzed based on considering three optical characteristics, reflection, transmission and absorption coefficient. From the calculation of the optical characteristics, the occurrence of the microcalcification in a digital mammogram was doubly confirmed. The results are further analyzed to satisfy the condition for the conservation of total energy that is the sum of reflection, transmission and absorption coefficient equals to one. For the obtained results, statistical features such as mean, median, mode, min, max, area, skewness, kurtosis and standard deviation are extracted and analyzed. By correlating the reflection, transmission, and absorption coefficient values of the binned image, the prediction class to categorize the considered mammogram image (malignant and normal) was also achieved with better classification accuracy. It is observed that SVM classifiers gives better accuracy in classification than KNN and Naïve Bayes classifiers. As a result, the proposed approach is found to be much more effective and efficient compared with the other existing classification approaches that have been used so far.

\section{Future works:}

As an extension of this work, we can introduce mass density as an additional optical characteristic for the detection of the size of microcalcification in the digital mammogram images. This can also be correlated with signal processing techniques for the analysis of the frequency and time. Transform methods can be applied to the digital images for the analysis of tumor tissue from the background tissue.

\section{REFERENCES}

1. C.Abirami, R.Harikumar,' Performance Analysis And Detection Of Micro Calcification In Digital Mammograms Using Wavelet Features', Ieee Wispnet 2016 Conference.

2. Ancy C A And Lekha S Nair,'An Efficient Cad For Detection Of Tumour In Mammograms Using Svm', International Conference On Communication And Signal Processing, April, 2017.

3. Jinshan Tang, Yongyi Yang,' Computer-Aided Detection And Diagnosis Of Breast Cancer With Mammography: Recent Advances', Ieee Transactions On Information Technology In Biomedicine, Vol 13, No. 2, March 2009

4. P. S. Vikhe, V. R. Thool,' Contrast Enhancement In Mammograms Using Homomorphic Filter Technique', 2016.

5. Y.Ratna Kumar, 'Measure Of Reflection And Transmission Coefficients For Bone Tissues', International Journal Of Innovative Research In Electrical, Electronics, Instrumentation And Control Engineering Vol. 3, Issue 1, January 2015.

6. R.Jemila Rose, S.Allwin,' Computerized Cancer Detection And Classification Using Ultrasound Images: A Survey', International Journal Of Engineering Research And Development,2013.

7. R.Jeba Christilda, G.R.Jothilakshmi,' Identification Of Cyst Through Its Optical Characteristics Using Sono Mammogram Images', Advances In Natural And Applied Sciences ,2017.

8. G.R. Jothilakshm , P. Mohana Priya,' Study Of Hardware Implementation On Size Of The Microcalcification Detection Using Embedded Systems', International Journal Of Engineering \& Technology,2018.

9. G. R. Jothilakshm, Arun Raaza,' Pattern Recognition And Size Prediction Of Microcalcification Based On Optical Characteristics

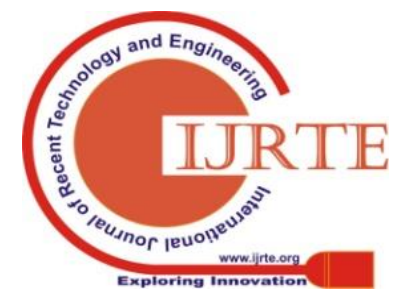


By Using Digital Mammogram Images', Society For Imaging Informatics In Medicine 2018.

10. Jaime S. Cardoso,' Mass Segmentation In Mammograms: A CrossSensor Comparison Of Deep And Tailored Features',2017.

11. Narain Ponraj, Jenkin Winston,' Novel Local Binary Textural Pattern For Analysis And Classification Of Mammogram Using Support Vector Machine', International Conference On Signal Processing And Communication (Icspc'17) -July 2017.

12. Jaya Sharma, R. P. Tewari,' A Combined Watershed Segmentation Approach Using K-Means Clustering For Mammograms', 2015 2nd International Conference On Signal Processing And Integrated Networks (Spin).

13. Juan Wang And Yongyi Yang,' Context-Sensitive Deep Learning For Detection Of Clustered Microcalcifications In Mammograms', 2018.

14. Katsushi Ikeuchi,'Determining Reflectance Properties Of An Object Using Range And Brightness Image',Ieee Transactions On Pattern Recognition And Machine Intelligence, November 1991.

15. B. Elizabeth Caroline, N.Vaijayanthi,' Feature Extraction Of Digital Mammogram Based On Multidimensional Complete Ensemble Empirical Mode Decomposition With Adaptive Noise', International Journal Of Advanced Engineering Technology,2016.

16. Songyang Yu, Ling Guan,' A Cad System For The Automatic Detection Of Clustered Microcalcifications In Digitized Mammogram Films', Ieee Transactions On Medical Imaging, Vol. 19, No. 2, February 2000.

17. Aya F. Khalaf, Inas A. Yassine,' Spectral Correlation Analysis For Microcalcification Detection In Digital Mammogram Images',2015.

18. Dae Hoe Kim, Jae Young Choi,' Region Based Stellate Features For Classification Of Mammographic Spiculated Lesions In ComputerAided Detection',2012.

19. Dr. S.A.Patil, M. B. Kuchanur,' Lung Cancer Classification Using Image Processing', International Journal Of Engineering And Innovative Technology (Ijeit) Volume 2, Issue 3, September 2012.

20. El-Sharkawy,'Breast Cancer Detection By Determination Of Optical Properties Of Nonmalignant And Malignant Breast Tissues', Journal Of Cancer Science And Clinical Oncology,2016.

21. Petter Jelle, 'Solar Radiation Glazing Factors for Window Panes, Glass Structures and Electrochromic Windows in Buildings Measurement and Calculation', 2012.

22. Brian Jiang, 'Optical properties of normal and cancerous human skin in the visible and near-infrared spectral range', 2006.

23. Katsunori Ishii, 'Determination of the tumor tissue optical properties during and after photodynamic therapy using inverse Monte Carlo method and double integrating sphere between 350 and $1000 \mathrm{~nm}$ ', 2011.

24. V G Peters, ' Optical properties of normal and diseased human breast tissues in the visible and near infrared', 1990.

25. Honda N, 'Optical properties of tumor tissues grown on the chorioallantoic membrane of chicken eggs: tumor model to assay of tumor response to photodynamic therapy' 2015.

\section{AUTHORS PROFILE}

Mrs.R.J.Hermalatha, is currently working as Head \& Assistant Professor in Biomedical Engineering Department in Vels Institute of Science Technology \& Advanced Studies- Chennai. She has 14 years of Teaching and research Experience.

Ms.T.R.Thamizhvani. , is currently working as Assistant Professor in Biomedical Engineering Department in Vels Institute of Science Technology \& Advanced Studies-Chennai. She completed her B.E Biomedical Engineering in Jerusalem College of Engineering \& M.E. Medical Electronics in SSN College of Engineering-Chennai. She has 2.5 years of experience in teaching \& research field.

Ms.Josephin Arockia Dhivya, is currently working as Assistant Professor in Biomedical Engineering Department in Vels Institute of Science Technology \& Advanced Studies-Chennai. She completed her B.Tech Biomedical Engineering \& M.Tech. Medical Instrumentation in Sathyabama Institute of Science \& Technology. She has 2.5 years of experience in teaching \& research field

Ms.A.Akalya is currently pursuing final year B.E. Biomedical Engineering in Vels Institute of Science Technology \& Advanced StudiesChennai.

Ms.R.Jeyanthi is currently pursuing final year B.E. Biomedical Engineering in Vels Institute of Science Technology \& Advanced StudiesChennai. 